appearance of the varicella rash in a 26-year-old male reported from the University of Geneva, Switzerland. A distal weakness of arms rapidly extended to the legs and was followed in 3 days by a severe bilateral flaccid paralysis. Sensation was normal. DTRs were present and plantar responses were absent. $\mathrm{CSF}$ on admission contained 4 cells $/ \mathrm{mm} 3$ and $0.61 \mathrm{~g} / 1$ protein; 1 week later, 17 cells and $2 \mathrm{~g} / 1$ protein. Serum anti-VZV IgG was 1:800 on admission and 1:1600 after 1 week. EMG showed an acute axonal motor polyneuropathy; 6 weeks later, there were fibrillation potentials and signs of neurogenic atrophy. Nerve conduction velocities showed no conduction block. Slow recovery and signs of reinnervation followed plasmaphareses, but a motor deficit persisted at 15 month follow-up. (Picard F, Gericke CA, Frey M, Collard M. Varicella with acute motor axonal neuropathy. Eur Neurol July 1997;38:68-71). (Respond: Dr Fabienne Picard, Clinique de Neurologie, Hopital Cantonal Universitaire de Geneve, 24, rue Micheli-du-Crest, CH-1211 Geneve 14, Switzerland).

COMMENT. Despite the cytoalbuminologic dissociation in the CSF, characteristic of a demyelinating Guillain-Barre syndrome, the EMG showed a primary motor axonal neuropathy without demyelination, and cranial nerves and DTRs were preserved. Campylobacter jejuni is the most frequent infectious agent causing motor axonal neuropathy, but this form of Guillain Barre syndrome may rarely be associated with varicella.

\title{
BRAIN MRI IN CONGENITAL MUSCULAR DYSTROPHIES
}

Magnetic resonance imaging (MRI) findings in 21 patients with congenital muscular dystrophy (CMD) complicated by cerebral anomalies were analysed from data collected at several Departments of Child Neurology, The Netherlands: Free University Hospital, and Emma Children's Hospital, Amsterdam; Sophia Children's Hospital, Rotterdam; Leiden University Hospital; Groningen University Hospital; and St Radboud University Hospital, Nijmegen. Four subgroups of CMD were defined by MRI characteristics: Grp 1, WalkerWarburg syndrome (WWS) (5 cases), CMD with retardation, macrocephaly, hydrocephalus, and eye abnormalities, showed posterior fossa abnormalities, aqueductal stenosis, polymicrogyria, and cerebral white matter demyelination on MRI; Grp 2, less severe form of muscle-eye-brain disease than WWS (4 patients), with normal or only focal white matter changes, less severe cerebral cortical dysplasia, frequent myopia, and similar posterior fossa abnormalities; Grp 3, CMD with isolated occipital agyria (3 patients), normal intelligence, no eye abnormalities, white matter changes, and hypoplasia of pons and vermis; and Grp 4, classic merosin-deficient CMD (MD-CMD) (8 patients), with diffuse cerebral white matter abnormalities only. (van der Knaap MS, Smit LME, Barth PG, et al. Magnetic resonance imaging in classification of congenital muscular dystrophies with brain abnormalities. Ann Neurol July 1997;42:50-59). (Respond: Dr van der Knaap, Department of Child Neurology, Free University Hospital, PO Box 7057, 1007 MB Amsterdam, The Netherlands).

COMMENT. The MRI is important in the classification of patients with congenital muscular dystrophy, both in terms of prognosis and in genetic studies. Four subgroups may be defined: Walker-Warburg syndrome, muscleeye-brain disease, merosin-deficient CMD, including the classic form and that of Fukuyama, and a newly recognized subtype with occipital agyria. 